\title{
Discussion on strengthening laboratory management and improve laboratory efficiency
}

\author{
Yining Liu $^{1}$ \\ ${ }^{1}$ School of Humanities , Changchun University of Technology ,Changchun 130012, China \\ aemail: 37495904@qq.com
}

Keywords: Laboratory management; Improve laboratory efficiency; Resource sharing

\begin{abstract}
Laboratory is the main component of the national science and technology innovation system, and it plays a more and more important role in the discipline, teaching, scientific research and personnel training in universities. Based on the analysis of the connotation and system of laboratory management, the overall planning of the laboratory management is put forward. Aiming at the main problems existing in the laboratory management in universities, this paper puts forward some suggestions on the construction and management of laboratory in the aspects of resource sharing, team building, and safety and so on. Laboratory management is conducive to optimize the allocation of resources. It provides a reliable guarantee for personnel training and scientific research.
\end{abstract}

\section{Introduction}

Laboratory is the main component of the national science and technology innovation system, which played a major role in university disciplines, teaching, scientific research and personnel training. It is an important sign of the level of running school and school strength. As Feng Duan academician said "the laboratory is the heart of modern universities" ${ }^{[1]}$. The status of the laboratory is indeed important, but the problem existence in Colleges and Universities of laboratory construction is very prominent, seriously affecting the laboratory construction and development. Therefore, it is necessary to study and discuss on it.

\section{Pay more attention to the overall planning of laboratory construction}

Schools need to strengthen the guidance of laboratory construction planning, and establish of the corresponding planning leading group ${ }^{[2]}$. Taking the key laboratory, laboratory teaching, public resources platform rely on each other, common development as the construction ideas, and taking the size of the school, the development direction, professional setting, subject construction and research direction as the basis, from construction funds, team building and other aspects of comprehensive consideration, to develop a suitable for the school, the laboratory's construction plans and blueprints, focus on highlighting their own characteristics, so that the resources to play a greater efficiency. Needs to be emphasized is that the construction of teaching laboratory in Colleges and universities can basically be planned and constructed in accordance with the teaching task, cultivation objectives, and key laboratory can also be planned and constructed in accordance with the provisions of the management. Subject laboratory and project based laboratory, this part of the laboratory is a kind of incubator which can inspire innovative thinking and give birth to achievement, is the enhance seedling for the next step. It has changed the status of teacher's self -management, let it develop freely and there is no unified planning and unified evaluation system. It improves the level of resource sharing platform, and realizes the sharing of resources.

\section{The sharing of resources, improve efficiency in the use of instruments and equipment}

In recent years, through extensive research and in-depth discussion, combined with the school 
actual, how to solve the problem of open sharing of large-scale instruments and equipment, put forward a set of innovative ideas, the establishment of a new funding mechanism, strengthen the construction of public resources platform, open fund, set up performance appraisal system and incentive mechanism and Interactive development with the experimental technical team, etc. Tightly around the goal of school development, from the reality of school, adhere to the ideas of overall planning, policy guidance, pilot run, step by step, the principles of relying on the subject, the relative concentration, charge sharing, resource sharing, take management system innovation and operation mechanism as a breakthrough, to format a scientific allocation, reasonable layout, multi-level, high efficiency, open style resource sharing system.

Prevent repeat purchase from the source

Around the "211 Project" and "985 Project" and other major construction projects, According to the platform and the discipline construction plan, large scale instrument and equipment disposition plan, combine with existing equipment resources, the scientific demonstration given by the leading group and the discipline group of large scale instrument and equipment management, finally decided the funding plan ${ }^{[3]}$. The demonstration, in addition to demonstrate its necessity, feasibility, advanced nature, we must fully consider the open sharing, from the source to reduce duplication of purchase, improve investment efficiency.

Intensify the construction of public resources platform

According to the school overall development goal and the discipline development plan, overall planning. scientific input, strengthen the construction of public resources platform, concentrated financial resources to build a number of large-scale equipment centers with relative concentration function and advanced equipment, promote universal resource sharing between disciplines, to meet the need of scientific and technological innovation. Public resources platform at university level were constructed based on demonstration construction, relatively independent operation. At the same time, the use of the new campus construction opportunity, combined with the old and new campus planning, to promote the construction of public resources platform at College level. Encourage and promote qualified college or between colleges to establish of large-scale instrument and equipment public resources platform at college level, shared use ${ }^{[4]}$. Public resources platform at college level was managed by college, the university to implement macro management.

Set up open fund

Set large-scale equipment sharing fund in the school, used for the financing of large equipment to open service on campus and support our school scientific research project. For different situations, open fund given different support (Figure 1), so that the limited funds can play its reasonable effect. On the fundamental research fund, open fund given key priority support.

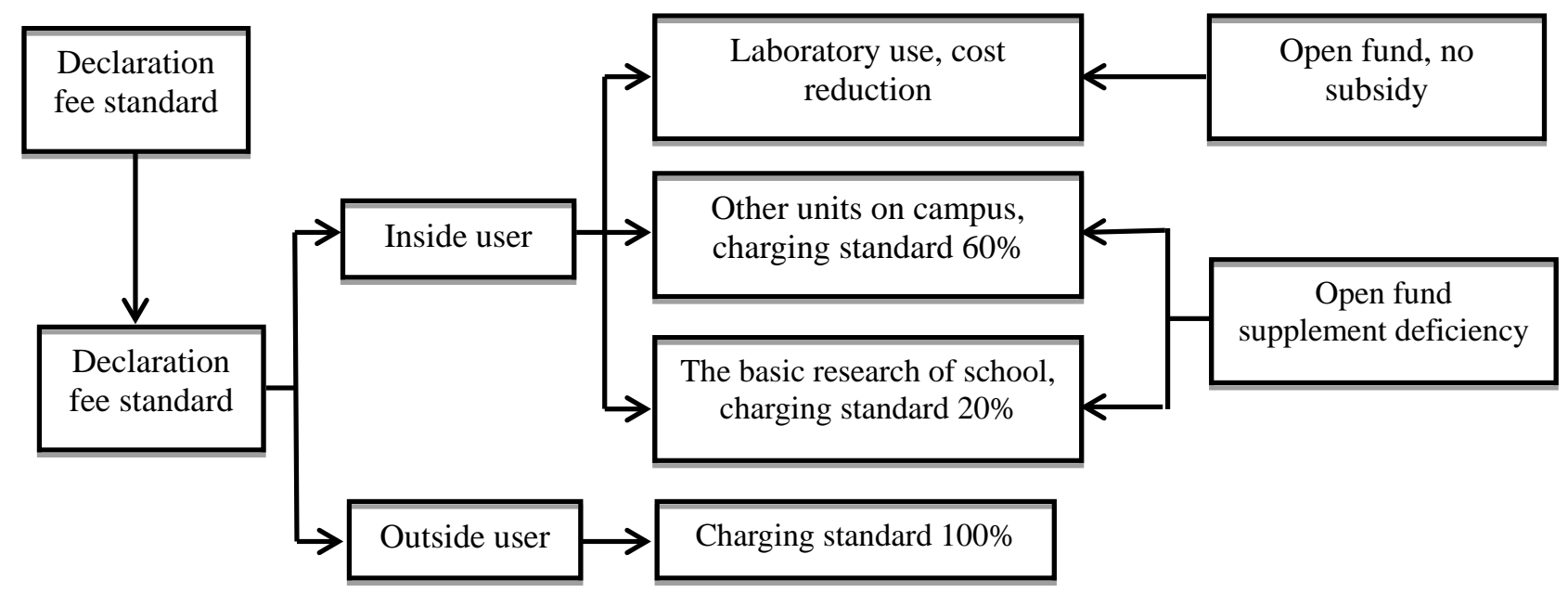

Fig.1 Flow chart of open service charge and fund for large scale instruments and equipment

Reasonable allocation

Open sharing of large equipment can try paid services, scientific pricing, unified fee and reasonable allocation, to encourage large-scale equipment initiative opening, realize the machine 
feed machine step by step, forming a virtuous circle. Open service charge can be used for equipment operation and maintenance and motivating people initiative and enthusiasm.

Establish network information platform of resource sharing for school

Establish a network information platform consist of the cooperative network of large-scale equipment sharing, large equipment resource database and information management system. To provide an unified and standardized information resources for internal and external users, online service, online management. At the same time, make full use of the relevant resource sharing platform of countries have established, such as the large scientific instruments sharing network of Northwestern, Shanxi Precision instruments and equipment sharing network, etc. Increase the open radiation intensity for the province, northwest and national.

The organic combination of resources sharing and the construction of experimental team

People are one of the key factors for open sharing of large equipment. The technology research and exploration, the function development and utilization of equipment, the high level of open service and so on, are in need of high-quality laboratory technicians. Must be organic combine of resources sharing and the construction of experimental team, to form the interactive development, ensure the open sharing of large equipment can really carry out for a long time. At present, the school has issued some suggestions on strengthening the construction of professional research and experimental technical team in Northwestern Polytechnical University ${ }^{[5]}$. By setting up post scientifically, to establish of new mechanism of professional and technical post assessment for full-time research and experimental technical team, establish scientific and perfect assessment methods, set up the experimental teaching achievement award and experimental technology award and other measures, to construct a reasonable structure, high quality and high level professional research and experimental technology ranks.

Establish the performance appraisal system and the reward and punishment mechanism

Comprehensive evaluation for the annual benefit of large scale instruments and equipment in the whole school, the assessment system is compose of machine using, machine condition, open service, the function development and utilization, scientific research achievements, personnel training. Released the results of evaluation to the school, and fit into the college evaluation index, at the same time as the future investment and adjustment basis for the equipment. School set up a "large scale equipment and equipment use efficiency Award" for the assessment, give recognition and reward to the units and individuals who have made outstanding achievements. For the units that long-term management of poor, resulting in low utilization of equipment and even idle, through online publishing, incremental control, equipment allocation and other measures to be punished accordingly, to promote the rational allocation of equipment.

\section{Further strengthen the construction of experimental team}

We must attach great importance to the construction of experimental technical team, clear positioning, establish a sound mechanism, quantitative check, improve the training, to improve the overall quality inspection technical team. Therefore, we put forward the basic idea of the experimental technical team construction. "attractive talent, optimize the structure, focus on training, improve quality, strengthen the assessment, improve the machine System." Build a high level inspection team as the construction goal with a fixed and mobile, full-time and part-time combination, dedicated to experimental science, dedication, excellent technology, reasonable structure and stable."

(1) Attract talent, optimize the structure.

According to the situation of experimental technology team lack of talent, our school take various measures, the introduction of incentives policy, constantly absorbing professional and high level technical personnel added to the team to make the total number of teams to reach an appropriate scale, and gradually optimize the structure, maintain a proper proportion with teachers, the overall level has greatly improved.

(2) Pay attention to improve the quality of training, improve quality

The laboratory technicians should strengthen the training of the knowledge structure and 
professional to improve the capacity, to adapt to the requirements of the development of the school, the school should set up special fund to support the team training work.

(3) Strengthen the assessment, improve the mechanism.

The school according to different position and different nature of the work formulate the corresponding measures for the assessment. Through the examination to strengthen the management, establish the corresponding incentive exit mechanism. It is not only stable the high level team but also improve people benefit.

\section{Pay more attention to the laboratory work safety}

In accordance with the laboratory safety management ideas, "publicity and education is the prerequisite, system management is fundamental, security check is the key, safety management is a measure", the school has issued more than a dozen management regulations, which make the laboratory safety management has rules, and implement the safety responsibility system step by step. At the same time, the requirements of the school set up a person responsible for laboratory safety work, each laboratory and special equipment has identified a security responsible person, which has formed everyone's attention, responsibility to the people's safety work situation. Actively organize, implement and implement national security policies, to form of a variety of safe production laws and regulations of the general level of education activities, in particular, to seize the favorable opportunity of the "safe production", and popularize safety production laws and regulations in the whole school. Through the safety publicity and education, the school staff's safety awareness and the ability to prevent accidents were further enhanced.

\section{Conclusions}

Problems encountered in the construction of the laboratory are very much. These are some superficial views on the problems which engaged in laboratory management in recent years. Also hope that we can further strengthen the research on laboratory management, and make their due contribution to the construction of laboratory in universities.

\section{References}

[1] Xu G H, Strengthening the construction of the original innovation ability in Universities [J]. Chinese University Science \& Technology,2002(8):10-13.

[2] Fan T, Tang J, Chen H Y, et al. Construction of Laboratory Technician Teams for Environment Science and Engineering in Agricultural Universities[J]. Research \& Exploration in Laboratory, 2012.

[3] Li J. Analysis of Logistics Laboratory Construction in Universities[J]. Hebei Jiaotong Science \& Technology, 2006.

[4] Zhang Y, Li X, Du X. The connotation and construction ways of laboratory culture in colleges and universities[J]. Experimental Technology \& Management, 2011.

[5] Liu Y, Wu F, Chen L, et al. Construction and practice of laboratory safety management system in universities based on SSE[J]. Experimental Technology \& Management, 2015. 